\title{
Gm40600 suppressed SP 2/0 isograft tumor by reducing Blimp1 and Xbp1 proteins
}

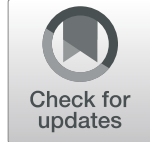

Ying Fang ${ }^{1,2}$, Ruonan $\mathrm{Xu}^{2,3}$, Bing Zhai ${ }^{2,4}$, Chunmei Hou' ${ }^{2}$ Ning Ma', Liang Wang ${ }^{3}$, Gencheng Han², Zhenyu Jiang $^{1 *}$ and Renxi Wang ${ }^{2^{*}}$ (i)

\begin{abstract}
Background: Multiple myeloma (MM), characterized by cancerous proliferation of plasmablasts (PB) and plasma cells $(P C)$, remains incurable in many patients. Differentially expressed molecules between MM PCs and healthy PCs have been explored in order to identify novel targets for treating MM. In the present study, we searched for novel MM therapeutic targets by comparing mRNA expression patterns between the Mus musculus myeloma plasmablastlike SP 2/0 cell line and LPS-induced PB/PC.

Methods: Gene expression profiles of LPS-induced PB/PC and SP 2/0 cells were determined using RNA-sequencing. A predicted gene (Gm40600) was found to be expressed at a low level in SP 2/0 cells. To study the role of Gm40600 in malignant PC, Gm40600 cDNA was cloned into a lentiviral vector (LV201) containing a puromycin selectable marker that was then transfected into SP 2/0 cells. Stable Gm40600-expressing SP 2/0 cells were selected using puromycin. The effect of Gm40600 on SP 2/0 cell proliferation, cell cycle/apoptosis, and tumor progression was assessed by cell counting kit-8 (CCK8), flow cytometry (FACS), and the SP 2/0 isograft mouse model, respectively. The effect of Gm40600 on mRNA and protein expression was evaluated by RNA-sequencing and western blotting, respectively.
\end{abstract}

Results: We found that SP 2/0 cells expressed lower level of Gm40600 mRNA as compared to LPS-induced PB/PC. Overexpression of Gm40600 significantly suppressed SP 2/0 cell proliferation and isograft tumor progression in an isograft mouse model by promoting apoptosis. In addition, Gm40600 overexpression suppressed transcription of the gene encoding Bcl2. Gm40600 overexpression also reduced the expression of PC-associated transcription factors Blimp1 and Xbp1, which promote transcription of the gene that encodes Bcl2.

Conclusions: Gm40600 reduced SP 2/0 cell proliferation and isograft tumor growth and progression by suppressing Blimp1 and Xbp1-mediated Bcl2 transcription to induce apoptosis. Thus, regulation of a human homolog of Gm40600, or associated factors, may be a potential therapeutic approach for treating MM.

Keywords: Gm40600, Plasma cells, Multiple myeloma, SP 2/0 cells

\section{Background}

Each year, deaths due to multiple myeloma (MM) with malignant plasma cells (PC) [1] is approximately $1 \%$ of all cancer deaths $[2,3]$. This is mainly because of the following two reasons: (1) MM is still incurable in many patients $[4,5]$; (2) some patients are refractory to current

\footnotetext{
*Correspondence: jlujzy@yahoo.com.cn; wang_renxi@hotmail.com 'Department of Rheumatology, First Hospital of Jilin University, Changchun 130021, China

${ }^{2}$ Laboratory of Immunology, Institute of Basic Medical Sciences, P.O. Box 130 (3), Taiping Road \#27, Beijing 100850, China

Full list of author information is available at the end of the article
}

conventional drugs $[6,7]$. Thus, it is urgent to find novel therapeutic way to treat MM [6].

Several reports have shown that MM PC share many common characteristics with healthy PC $[2,3,8]$. Normally, the differentiation of activated $\mathrm{B}$ cells into $\mathrm{PC}$ is strictly controlled by several genes, including B cellassociated Pax5, germinal center (GC) B cell-associated Bcl6, and PC-specific genes such as IRF4, Prdm1 (Blimp1), and Xbp1 $[9,10]$. Bcl2 also promotes B cell differentiation into $\mathrm{PC}$, as $\mathrm{Bcl} 2$ with anti-apoptotic activity supports GC B cells and PC survival [11, 12].

(c) The Author(s). 2019 Open Access This article is distributed under the terms of the Creative Commons Attribution 4.0 International License (http://creativecommons.org/licenses/by/4.0/), which permits unrestricted use, distribution, and reproduction in any medium, provided you give appropriate credit to the original author(s) and the source, provide a link to the Creative Commons license, and indicate if changes were made. The Creative Commons Public Domain Dedication waiver (http://creativecommons.org/publicdomain/zero/1.0/) applies to the data made available in this article, unless otherwise stated. 
As cancerous cells, MM PC express MM-specific molecules that differ from healthy PCs [13-15]. To explore novel MM therapeutic targets, it is necessary to identify molecules that differ between healthy PC and MM PC. Because we found that Mus musculus myeloma PB-like SP 2/0 cells (MM PB/PC) expressed a significantly lower level of Gm40600 (a predicted gene) mRNA as compared to LPS-induced $\mathrm{PB} / \mathrm{PC}$ (normal $\mathrm{PB} / \mathrm{PC}$ ), the effect of $\mathrm{Gm} 40600$ on SP 2/0 cell growth was tested.

\section{Methods}

\section{Mice}

$\mathrm{Balb} / \mathrm{c}$ and $\mathrm{CD} 19^{\text {cre }}$ mice have been previously described $[16,17]$. The Floxed Stch $\left(\operatorname{Stch}^{\mathrm{f} / \mathrm{f}}\right)$ mice in a B6 background were generated by Shanghai Biomodel Organism Science \& Technology Development Co.,Ltd. (Shanghai, China). Stch ${ }^{\mathrm{f} / \mathrm{f}}$ mice were crossed with $\mathrm{CD} 19^{\text {cre }}$ mice to delete Stch in B cells. Gm40600 transgenic mice (cat no. TGB180522CEI02) were purchased from Cyagen Co., Ltd. (Guangzhou, China).

\section{RNA-sequencing}

B220 ${ }^{+}$B cells were sorted from splenocytes of 7- to 9week female Balb/c, Stch ${ }^{\mathrm{f} / \mathrm{f}}$, and $\mathrm{CD} 19^{\mathrm{cre}} \mathrm{Stch}^{\mathrm{f} / \mathrm{f}}$ mice $(3$ mice per group) using B220 microbeads (Cat No. 130049-501, Miltenyi Biotec, Germany), B220 ${ }^{+}$B cells were stimulated with $10 \mu \mathrm{g} / \mathrm{ml}$ LPS (L2630, Sigma, St Louis, $\mathrm{MO}$ ) for 3 days in vitro as previously described [18]. SP $2 / 0$ cells (ATCC ${ }^{\ominus}$ CRL-1581, Rockville, MD, USA) were thawed, passaged three times, and then cultured for 2 days in fresh medium. RNeasy Mini Kit (Qiagen, Venlo, Netherlands) was used to isolate and purify total RNA from cells. NanoDrop ${ }^{\circ}$ ND-1000 spectrophotometer and Agilent 2100 Bioanalyzer and RNA 6000 NanoChips (Agilent, Palo Alto, CA, USA) were used to determine RNA concentration and quality, respectively. TruSeq Stranded Total RNA Library Prep Kit with Ribo-Zero Gold (Illumina) was used to prepare Libraries. Transcripts were analyzed by RNA-sequencing (Genewiz Corp., Suzhou, China) using a standard method [18].

\section{qPCR analysis}

Total RNA was extracted from Vector- or Gm40600expressing SP $2 / 0$ cells, and LPS-stimulated $\mathrm{PB} / \mathrm{PC}$ with Trizol (Invitrogen Life Technologies). qPCR has been employed using a previous method [18] to quantify mouse Gm40600 gene expression. GAPDH mRNA expression is used to normalized relative mRNA expression that is then calculated relative to mRNA in SP $2 / 0$ cells (set to 1 ).

\section{Effect of Gm40600 on SP 2/0 growth}

Gm40600 cDNA (accession: XM_011243239) was synthesized by General Biosystems Corp. (Anhui, China) and subcloned into LV201 (Fugene Corp., Guangzhou, China), a lentiviral vector with a puromycin selectable marker. Gm40600-expressing LV201 or control LV201 (empty vector) were transfected into SP $2 / 0$ cells using a previously described method $[18,19]$. Puromycin $(10 \mu \mathrm{g} /$ $\mathrm{ml}$; Sigma) was used to select stable transfectants. Counting kit-8 (CCK8), a propidium iodide (PI)/FACS, and the SP 2/0 isograft mouse model were previously described $[18,20,21]$ and used to assess the effect of Gm40600 on SP 2/0 cell proliferation, cell cycle, and isograft tumor progression, respectively.

\section{Gm40600 localization and protein expression}

Gm40600 was cloned into EGFP-expressing LV122 (Fugene Corp., Guangzhou, China) to express a Gm40600-EGFP fusion protein. Gm40600-EGFPexpressing LV122 was transfected into SP 2/0 cells using a previously described method [18]. Gm40600-EGFP protein localization was analyzed by immunofluorescence and confocal microscopy according to a previously described method [18]. The effect of Gm40600 on Bcl6, IRF4, Blimp1, Xbp-1, Trp53, and Bcl2 was determined by western blotting using a previously described method [18]. The western blots were probed with rabbit against mouse Bcl6 (sc-858, Santa Cruz Biotech), IRF4 (ab104803), Blimp1 (sc-25,380, Santa Cruz Biotech), Xbp-1 (ab37152, Abcam), Trp53 (\#2524, Cell Signaling Tech), Bcl2 (ab59348, Abcam) and $\beta$-tubulin (KM9003T, SunGene Biotech) antibodies.

\section{$\mathrm{Bcl} 2$ promoter reporting gene analysis}

Lv81/Blimp1 (0.5 $\mu \mathrm{g}$, cat\# EX-Mm24401-Lv81, Fugene Corp., Guangzhou, China), pEZX-PG04.1/Bcl2 promoter $(0.5 \mu \mathrm{g}$, cat\# MPRM19639-PG04, Fugene Corp., Guangzhou, China), and pRLSV-40 vector $(0.05 \mu \mathrm{g}$, cat\# E2231, Promega Corp.) were co-transduced into $4 \times 10^{5}$ $293 \mathrm{~T}$ cells according to our previous study [18]. In some experiments, firefly luciferase reporter plasmid pEZXPG04.1/Bcl2 promoter and renilla luciferase reporter vector pRLSV-40 vector were transduced into stable Gm40600- or vector-expressing SP 2/0 cells. On day 3, 1420 Multilabel Counter (1420 Victor 3, PerkinElmer Corp.) was used to determine the activity of firefly and renilla luciferase.

\section{Determination of $\lg M, \lg G 1$, and $\lg G 2 a$ antibody levels by ELISA}

B cells from WT and Gm40600 transgenic mice were stimulated for 3 days in vitro with $10 \mu \mathrm{g} / \mathrm{ml} \mathrm{LPS}$. Antibody levels of in the supernatant were determined using mouse IgM, IgG1, and IgG2a ELISA kits (eBiosciences, Cat\# 88-50,470-86, 88-50,140-22, and 88-50,420-88, respectively) as the instructions of the manufacturers. 


\section{Statistics}

Cellular apoptosis, tumor volume, and tumor weight were compared between the vector alone group and the Gm40600 overexpressing group using the Student's $t$ test. The change of cell proliferation at three different time points and the percentage of three different cell cycles phases (G1, S, and G2) were compared between the vector alone group and the Gm40600 overexpressing group using two-way ANOVA analysis. SEM was used as mean \pm standard error of the mean. $p<0.05$ value was considered to be statistically significant.

\section{Results}

\section{SP 2/0 cells express low levels of Gm40600 mRNA}

Plasmablasts (PB) induced by LPS $[20,22]$ and the Mus musculus myeloma PB-like SP $2 / 0$ cells were used as normal $\mathrm{PB} /$ plasma cells $(\mathrm{PC})$ and $\mathrm{MM} \mathrm{PB} / \mathrm{PC}$, respectively. Both LPS-stimulated $\mathrm{PB} / \mathrm{PC}$ and $\mathrm{SP} 2 / 0$ cells expressed high levels of mRNA corresponding to the following $\mathrm{PB} / \mathrm{PC}$-associated transcription factors IRF4, Prdm1 (Blimp1), and Xbp1 (Table 1). Interestingly, as compared to LPS-stimulated PB/PC, SP 2/0 cells expressed a significantly lower level of Gm40600 mRNA (Table 1). Thus, reduction of Gm40600 may promote malignant growth of SP 2/0 cells.

\section{SP 2/0 cell proliferation was suppressed by $\mathrm{Gm} 40600$}

To explore the effect of Gm40600 in malignant growth of SP 2/0 cells, an LV201 construct expressing Gm40600 were transfected into SP 2/0 cells. Stable transfectants expressing Gm40600 or Vector were selected by puromycin. qPCR assay demonstrated that Gm40600 mRNA was significantly increased in stably transfected SP $2 / 0$ cells and lower than that in LPS-induced PB/PC (Additional file 1: Figure S1). Critically, CCK8 assay showed that cell number is time-dependently lower in

Table 1 SP 2/0 cells express low level of Gm40600 mRNA

\begin{tabular}{llllll}
\hline \multirow{2}{*}{$\begin{array}{l}\text { Gene } \\
\text { Symbol }\end{array}$} & \multicolumn{2}{l}{ Total exon fragments } & & \multicolumn{2}{l}{ FPKM } \\
\cline { 2 - 3 } \cline { 5 - 6 } & LPS & SP 2/0 & & LPS & SP 2/0 \\
\hline Pax5 & 11,625 & 70 & & 34.34 & 0.71 \\
Bach2 & 3318 & 0 & & 7.92 & 0 \\
Bcl6 & 403 & 230 & & 4.25 & 5.59 \\
Irf4 & 9191 & 5272 & & 12.6 & 90.41 \\
Prdm1 & 6055 & 1233 & & 32.43 & 18.83 \\
Xbp1 & 15,347 & 8127 & & 196.23 & 293.8 \\
Gm40600 & 552 & 4 & 22.87 & 0.35 \\
\hline
\end{tabular}

$\mathrm{B}$ cells from the splenocytes of three Balb/c mice per group were sorted by B220 microbeads, and stimulated for 3 days in vitro by $10 \mu \mathrm{g} / \mathrm{ml}$ LPS. SP $2 / 0$ cells were thawed, passaged for 3 times and then cultured for 2 days in fresh medium. The transcripts in LPS-stimulated B cells and SP 2/0 cells were determined by RNA-sequencing. Total exon fragments and FPKM (FPKM = total exon fragments / mapped reads (millions) / exon length (KB), Fragments per Kilo bases per Million reads) values of selected genes encoding Pax5, Bach2, Bcl6, Irf4, Prdm1, Xbp1, and Gm40600 are shown
Gm40600-expressing SP 2/0 cells than that in vectorexpressing SP 2/0 cells (Fig. 1a). Furthermore, cell cycle and apoptosis analysis suggested that Gm40600 overexpression did not affect the cell cycle but it did promote cell apoptosis (Fig. 1b-d). Collectively, these results demonstrated that Gm40600 significantly suppressed proliferation of SP $2 / 0$ cells by promoting apoptosis.

\section{Overexpression of Gm40600 suppressed SP 2/0 isograft tumor progression}

The SP 2/0 isograft mouse model $[18,19]$ was used to assess the effect of Gm40600 on SP 2/0 isograft tumor growth. Gm40600-expressing SP $2 / 0$ cells $\left(5 \times 10^{6}\right.$ cells per mouse) were injected subcutaneously into 8 -week-old $\mathrm{Balb} / \mathrm{c}$ mice (5 mice per group). Tumor volumes were measured on day $0,8,9,10,11,12,13$, and 14 after the isograft. Consistent with in vitro data, Gm40600 significantly suppressed SP2/0 isograft tumor progression in a time-dependent fashion (Fig. 2a). Tumors were imaged on day 14 following isograft, which showed that SP 2/0 isograft tumor sizes were decreased by Gm40600 (Fig. 2b). The Gm40600-mediated reduction of the volumes and weights of SP 2/0 isograft tumors was statistically significant (Fig. 2c, d). Collectively, these data demonstrated that Gm40600 suppressed growth and progression of tumors in the SP 2/0 isograft tumor model.

\section{Gm40600 overexpression reduced Blimp1 and Xbp1 proteins in SP $2 / 0$ cells}

As shown above, Gm40600 overexpression promoted SP 2/0 cell apoptosis. Next, RNA-sequencing assay was used to determine which molecule is involved in Gm40600mediated apoptosis. Gm40600 had no effect on the mRNA levels of Pax5 (mature B cell-associated molecule), Bcl6 and Bach2 (GC B cell-associated molecules), IRF4, Prdm1 (Blimp1) and either unspliced or spliced Xbp1 (PC-associated molecules), Trp53 and Mcl1 (apoptosis-associated molecules) (Table 2). Interestingly, mRNA corresponding to the anti-apoptosis factor $\mathrm{Bcl} 2$ was reduced by Gm40600 (Table 2). These results suggest that Gm40600 affected $\mathrm{Bcl} 2$ transcription.

To explore the mechanism by which Gm40600 regulates transcription, the location of Gm40600 in SP 2/0 cells was examined. SP $2 / 0$ cells expressing either Gm40600-EGFP or EGFP alone were imaged on a GE IN Cell Analyzer 2000. The results demonstrated that EGFP alone was distributed throughout the cells, whereas Gm40600-EGFP was mainly localized to the cytoplasm (Fig. 3a). These results suggest that Gm40600 is not a transcription factor.

Previous studies have shown that Blimp-1 can induce the expression of Xbp-1 [23, 24], which up-regulates the expression of $\mathrm{Bcl} 2 \mathrm{mRNA}$ by directly binding ACTGCRE sites in the $\mathrm{Bcl} 2$ gene promoter $[25,26]$. Thus, we 


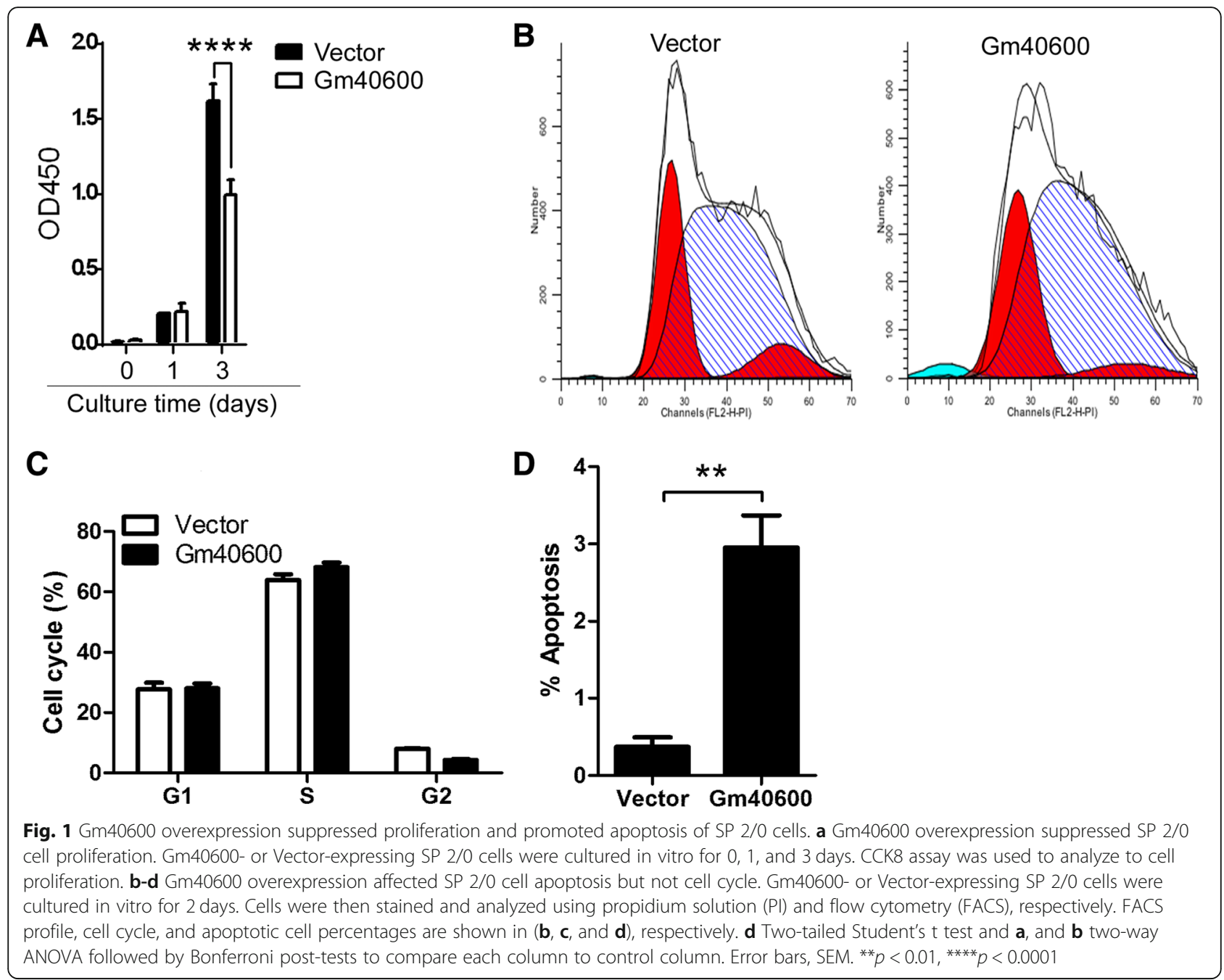

propose that $\mathrm{Gm} 40600$ affects $\mathrm{Bcl} 2$ mRNA expression via Blimp1 and Xbp1. As expected, we found that Gm40600 could reduce Blimp1 and spliced Xbp-1 ( 54 $\mathrm{kDa}$ ) protein in SP 2/0 cells (Fig. 3b, Additional file 1: Figure S2). Accordingly, Gm40600 also reduced expression levels of the anti-apoptosis protein Bcl2 (Fig. 3b) and $\mathrm{Bcl} 2$ promoter activation (Additional file 1: Figure S3) in SP 2/0 cells. In addition, Blimp1 significantly activated the $\mathrm{Bcl} 2$ gene promoter (Fig. $3 \mathrm{c}$ ). These results suggest that Gm40600 suppresses $\mathrm{Bcl} 2$ gene transcription by reducing Blimp1 and Xbp1 transcription factors.

\section{Gm40600 overexpression suppressed antibody production in LPS-stimulated primary B cells}

To study the role of Gm40600 in normal PC, we found that Stch (a gene coding the heat shock protein family A [Hsp70] member 13) was mainly expressed in PC and a B cell-specific knock-out of Stch did not affect B-cell mature and activation but reduced PC production (data not shown). In this work, Stch deficiency reduced LPS- induced $p r d m 1$ and $x b p 1$ mRNA by $50 \%$ (Table 3 ). Accordingly, LPS-stimulated $\mathrm{Stch}^{-1-} \mathrm{B}$ expressed almost no Gm40600 (Table 3). Collectively, these data suggest that Gm40600 expression may positively correlate with Prdm1 and Xbp1 expression and involved in nonmalignant PC generation/maintenance.

To further explore the function of Gm40600 in antibody production, Gm40600 transgenic mice were developed. LPS can effectively induce PC production and antibody secretion [20]. Thus, LPS was used to induce antibody production in B cells from wild-type (WT) and Gm40600 transgenic mice. As expected, Gm40600 overexpression suppressed antibody production in LPSstimulated primary B cells (Fig. 4).

\section{Discussion}

Untill now, the expression pattern of Gm40600 remains unclear and its function is uncharacterized. RNAsequencing demonstrated that LPS-induced $\mathrm{PB} / \mathrm{PC}$ expressed a high level of Gm40600 mRNA, whereas 


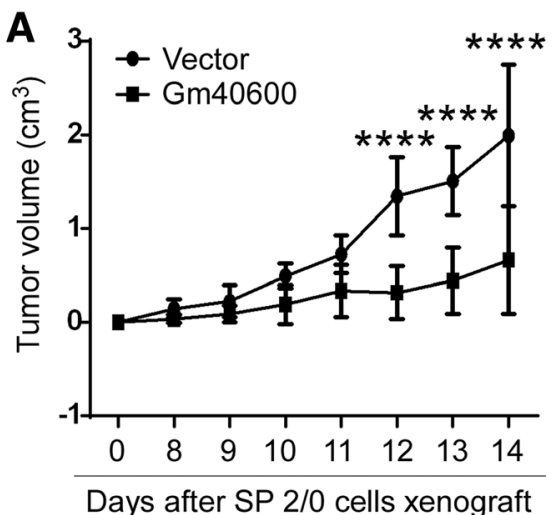

B

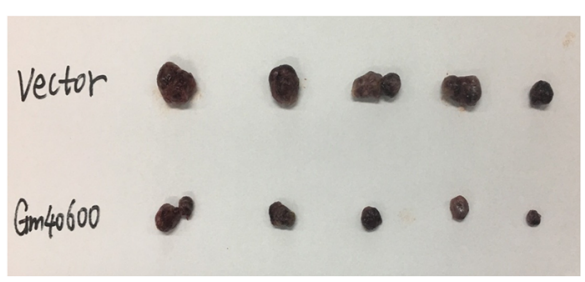

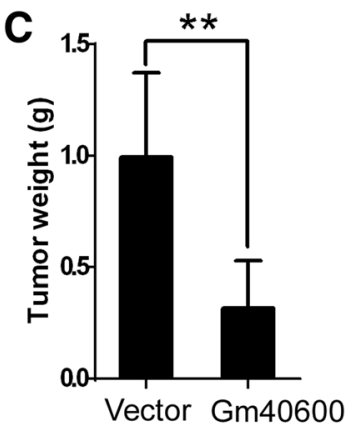

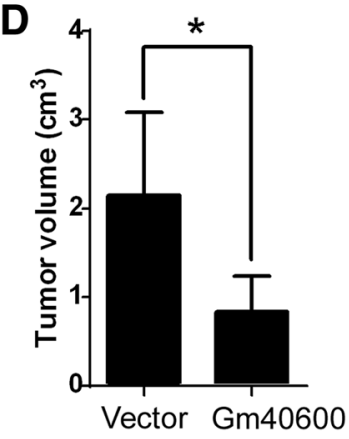

Fig. 2 Gm40600 overexpression suppressed tumor progression in the SP 2/0 isograft mouse model. Gm40600- or Vector-expressing SP 2/0 cells were injected subcutaneously into Balb/c mice. a Tumor volumes were determined on day 0, 8, 9, 10, 11, 12, 13, and 14 after isograft. On day 14 after the SP 2/0 isograft, images of subcutaneous tumor tissues (b), tumor weights (c), and tumor volumes (d) were recorded. $\mathbf{a}$, $\mathbf{c}$, and $\mathbf{d}$ Data from three independent experiments $(n=15)$. $\mathbf{b}$ Data from one experiment $(n=5)$. Error bars, SEM. Two tailed Student's t-test, ${ }^{*} P<0.05$, ${ }^{* *} p<0.01,{ }^{* * *} p<0.0001$

cancerous SP $2 / 0$ cells expressed a low level of Gm40600 mRNA (Table 1). In addition, Gm40600 was identified as a suppressive molecule that suppresses cancerous SP 2/0 cell proliferation (Fig. 1a) and isograft tumor progression (Fig. 2). With a high incidence of survival because of usage of these drugs such as bortezomib, thalidomide and lenalidomide [27, 28], MM is still incurable and novel therapeutic strategies are needed [28]. To explore potential therapies, more knowledge of MM biology is needed [28, 29]. This work demonstrated that cancerous SP 2/0 cells expressed very low levels of Gm40600 (Table 1), whereas Gm40600

Table 2 Gm40600 overexpression does not affect the mRNA expression of plasma cells-associated transcription factors and apoptosis-associated molecules

\begin{tabular}{|c|c|c|c|c|c|c|}
\hline \multirow[t]{2}{*}{ Gene Symbol } & \multicolumn{3}{|c|}{ Total exon fragments } & \multicolumn{3}{|l|}{ FPKM } \\
\hline & Vector & Gm40600 & Fold change & Vector & Gm40600 & Fold change \\
\hline Pax5 & 4.19 & 12 & 2.87 & 0.03 & 0.09 & 3 \\
\hline BCL6 & 171 & 127 & 0.74 & 2.55 & 2.07 & 0.81 \\
\hline Bach2 & 0 & 0 & & 0 & 0 & \\
\hline IRF4 & 14,695 & 12,090 & 0.82 & 163.91 & 147.68 & 0.9 \\
\hline Prdm1 & 2752 & 2584 & 0.94 & 31.65 & 33.71 & 1.07 \\
\hline Xbp1 (unspliced) & 7731.6 & 8027.07 & 1.04 & 190.96 & 217.1 & 1.14 \\
\hline Xbp1 (spliced) & 101.4 & 116.93 & 1.15 & 5.23 & 6.6 & 1.26 \\
\hline Trp53 & 4337 & 5386 & 1.24 & 127.9 & 173.83 & 1.36 \\
\hline $\mathrm{BCl} 2$ & 34 & 18 & 0.53 & 0.27 & 0.14 & 0.52 \\
\hline Mcl1 & 21,732 & 26,477 & 1.22 & 406.54 & 576.35 & 1.42 \\
\hline
\end{tabular}

The stable Gm40600- and Vector-expressing SP 2/0 cells were cultured for 2 days in fresh medium. The transcripts were determined by RNA-sequencing. Total exon fragments and FPKM (FPKM = total exon fragments / mapped reads (millions) / exon length (KB), Fragments per Kilo bases per Million reads) values of selected genes encoding Pax5, Bach2, Bcl6, Irf4, Prdm1, unspliced and spliced Xbp1, Trp53, Bcl2, and Mcl1, and the fold change of gene expression in Gm40600expressing SP $2 / 0$ cells to that in vector-expressing SP $2 / 0$ cells are shown 


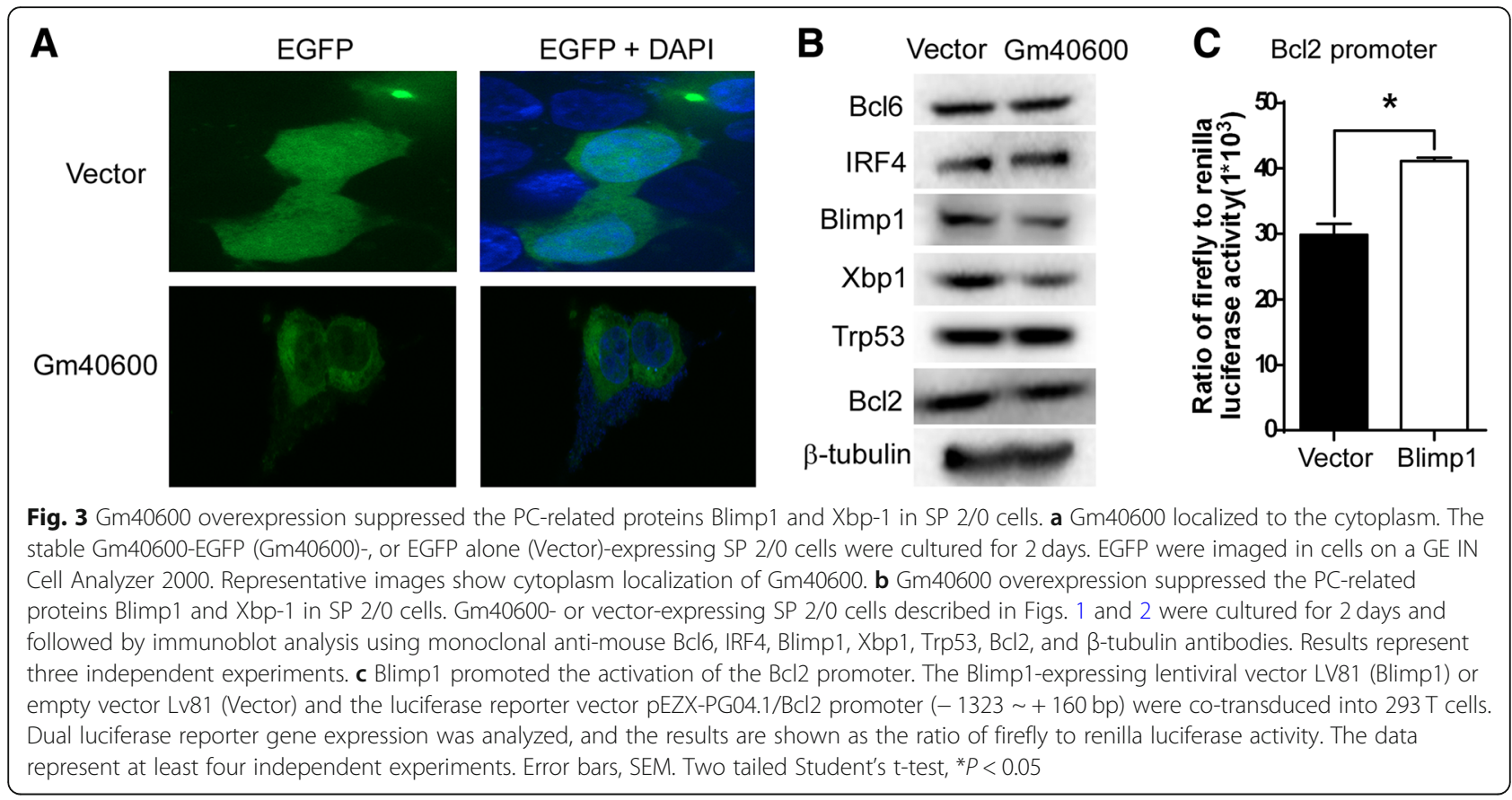

overexpression suppressed cancerous SP 2/0 cell growth (Figs. 1 and 2). In addition, Gm40600 mRNA was significantly lower in stably transfected SP $2 / 0$ cells than that in LPS-induced PB/PC (Additional file 1: Figure S1). These results suggest that overexpression of Gm40600 in SP $2 / 0$ cells is lower than its physiological expression in LPS-induced $\mathrm{PB} / \mathrm{PC}$. This supports that the downregulation of Gm40600 in SP 2/0 cells is a necessary part of tumor development. Thus, it is worth to identify a human homolog for $\mathrm{Gm} 40600$ and explore the role of a human homolog of Gm40600 in MM development.

Table 3 Stch deficiency reduced plasma cells and Gm40600 expression induced by LPS

\begin{tabular}{|c|c|c|c|c|}
\hline \multirow{2}{*}{$\begin{array}{l}\text { Gene } \\
\text { Symbol }\end{array}$} & \multicolumn{2}{|c|}{ Total exon fragments } & \multicolumn{2}{|l|}{ FPKM } \\
\hline & $\overline{S t c h}{ }^{f / f}$ & CD19 $9^{\text {cre }}$ Stch $^{f / f}$ & $\overline{S t c h} f / f$ & CD19 ${ }^{\text {cre }}$ Stch $^{f / t}$ \\
\hline Pax5 & 21,163 & 25,578 & 135.59 & 143.21 \\
\hline Bach2 & 2578 & 2608 & 8.17 & 7.22 \\
\hline Bcl6 & 660 & 590 & 6.38 & 4.99 \\
\hline Irf4 & 11,044 & 9245 & 81.82 & 59.86 \\
\hline Prdm1 & 2294 & 1288 & 17.02 & 8.35 \\
\hline Xbp1 & 6755 & 3460 & 176.95 & 79.21 \\
\hline Gm40600 & 391 & 14 & 25.96 & 0.81 \\
\hline
\end{tabular}

B220 $\mathrm{B}$ cells from 7 to 9-week-old CD19 $9^{\text {cre }}$ Stch $^{\text {f/f }}$ mice and their WT littermates $\left(\mathrm{Stch}^{\mathrm{f} / \mathrm{f}}\right.$ ) were stimulated for 3 days in vitro with $10 \mu \mathrm{g} / \mathrm{ml} \mathrm{LPS}$. On day 3 following LPS stimulation, the transcripts were determined by RNAsequencing. Total exon fragments and FPKM (FPKM = total exon fragments / mapped reads (millions) / exon length (KB), Fragments per Kilo bases per Million reads) values of selected genes encoding Pax5, Bach2, Bcl6, Irf4, Prdm1, Xbp1, and Gm40600 are shown
The survival of malignant PC [30], including MM cells [31, 32], was maintained by anti-apoptosis molecules. This inspired researchers to target these anti-apoptotic proteins as a strategy for treating MM [33]. Apoptosisinduced drugs such as Bortezomib have been approved to treat MM [34]. Consistent with these studies, we demonstrated here that Gm40600 overexpression induced SP 2/0 cell apoptosis (Fig. 1b-d).

An important regulator in cell apoptosis is Bcl-2 [35, 36]. Studies have shown that several human tumors aberrantly overexpress $\mathrm{Bcl}-2$, including $\mathrm{MM}[37,38]$. However, the mechanisms underlying aberrant $\mathrm{Bcl}-2$ expression remain largely unknown [39]. Our data demonstrated that $\mathrm{Gm} 40600$ reduced both Bcl 2 mRNA and protein in SP 2/0 cells (Table 2, Fig. 3b).

$\mathrm{Xbp} 1$ can effectively induce $\mathrm{Bcl} 2$ transcription by directly binding to CTG-CRE sites in the Bcl 2 gene promoter or indirectly by ESR1 [25, 26, 40]. Our work demonstrated that Gm40600 reduced Bcl2 mRNA (Table 2) by suppressing $\mathrm{Bcl} 2$ promoter activation (Additional file 1: Figure S3), and Xbp1 protein (Fig. 3b, Additional file 1: Figure S2) in SP $2 / 0$ cells. This suggests that Gm40600 suppresses Bcl2 transcription by reducing Xbp1 protein levels. Previous studies have shown that Blimp-1 induces expression of Xbp1 [41-43], which promotes antibody production [44, 45]. Our data showed that both Blimp1 and Xbp1 protein levels were reduced in SP 2/0 cells overexpressing Gm40600 (Fig. 3b, Additional file 1: Figure S2). Collectively, these results suggest that Gm40600 induced apoptosis by reducing Blimp1 and Xbp1-mediated Bcl2 transcription. 

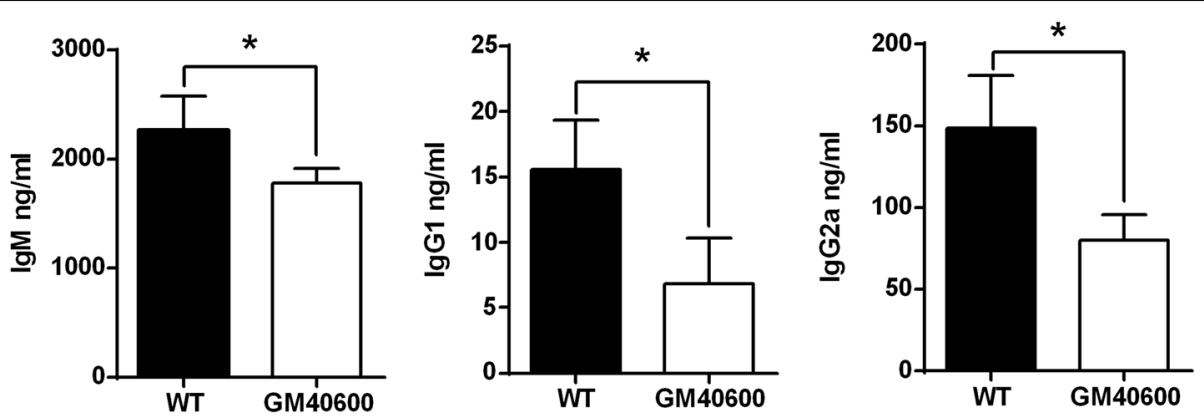

Fig. $4 \mathrm{Gm} 40600$ overexpression suppressed antibody production. $10 \mu \mathrm{g} / \mathrm{ml}$ LPS was used to stimulate B cells from the splenocytes of 7- to 9week-old WT and Gm40600 transgenic mice (three mice per group, total three independent experiments). On 3 days, lgM, lgG1, and lgG2b levels in cell culture supernatant was analyzed using ELISA assay. Error bars, SEM. Two tailed Student's t-test, ${ }^{*} P<0.05$

Blimp1 promotes the survival of PC in healthy donors and MM patients, whereas its deficiency causes PC apoptosis [31, 46]. In accordance with these studies, our data demonstrated that overexpression of Gm40600 reduced both Blimp1 expression (Fig. 3b) and promoted apoptosis (Fig. 1b-d). Thus, Gm40600 overexpression promoted SP $2 / 0$ cell apoptosis by reducing Blimp1 expression.

Finally, we found that Gm40600 expression is positively related to $\operatorname{Prdm} 1$ and $\mathrm{Xbp} 1$ expression involved in nonmalignant PC generation/maintenance (Table 3). However, Gm40600 overexpression suppressed antibody production in LPS-stimulated primary B cells (Fig. 4). Collectively, these data suggest that Gm40600 is a negative regulator of antibody production.

There are still some unsolved questions: 1 , the mechanisms by which Gm40600 reduces Blimp1; 2, the physiological function of Gm40600 in Prdm1 and Xbp1 expression, and nonmalignant PC generation/maintenance; 3 , whether there are human orthologs of the Gm40600 gene; 4, the pathogenic role of the human ortholog of Gm40600 gene in MM; 5, whether regulation of the Gm40600 human ortholog can be used to treat MM. Researching these questions will be important for understanding the role of $\mathrm{Gm} 40600$ and its human orthologs in MM.

\section{Conclusions}

Compared to LPS-induced PC, cancerous SP $2 / 0$ cells expressed a low level of Gm40600 mRNA. Overexpression of Gm40600 suppressed SP 2/0 cell proliferation and isograft tumor growth by reducing Blimp1 and Xbp1-mediated transcription of the anti-apoptosis molecule Bcl2. Thus, it is necessary to explore the role of a human homolog of Gm40600 in MM. This will guarantee that regulation of a human homolog of Gm40600, or associated factors, may represent a novel therapeutic strategy for treating MM.

\section{Additional file}

Additional file 1 : Figure S1. Gm40600 mRNA expression was significantly lower in stable Gm40600-expressing SP 2/0 cells than that in LPS-induced plasmablasts/plasma cells (PB/PC). B cells from the splenocytes of three 8-9-week-old Balb/c mice per group were sorted by B220 microbeads, and stimulated for 3 days in vitro by $10 \mu \mathrm{g} / \mathrm{ml}$ LPS. The stable Vector- or Gm40600-expressing SP 2/0 cells were thawed, passaged for 3 times and then cultured for 3 days in fresh medium. Gm40600 mRNA expression was determined by qPCR in Vector-expressing SP 2/0 cells (Vector-SP2/0), Gm40600-expressing SP 2/0 cells (Gm40600-SP2/0) and LPSstimulated PB/PC (LPS-PB/PC) were determined. Relative mRNA levels are normalized to GAPDH mRNA expression and calculated relative to the mRNA expression in SP 2/0 cells, set to 1. Figure S2. Gm40600 overexpression reduced Blimp1 and Xbp-1 protein in SP 2/0 cells. Vector- or Gm40600-expressing SP 2/0 cells were thawed, passaged for 3 times and then cultured for 3 days in fresh medium. The cells were then collected and subjected to immunoblot analysis with monoclonal anti-mouse antibodies for Xbp1, Blimp1, and $\beta$-tubulin. Results represent three independent experiments. Figure S3. Gm40600 overexpression suppressed the $\mathrm{BC} 2$ promoter activation in SP 2/0 cells. The luciferase reporter vector pEZX-PG04.1/Bcl2 promoter (- $1323 \sim+160 \mathrm{bp})$ and renilla luciferase reporter vector pRLSV-40 vector were co-transduced into stable Gm40600or vector-expressing SP 2/0 cells. The cells were cultured for 3 days. Dual luciferase reporter gene expression was analyzed, and the results are shown as the ratio of firefly to renilla luciferase activity. The data represent three independent experiments. Error bars, SEM. Two tailed Student's t-test, ${ }^{* *} P<0.01$. (PDF $\left.203 \mathrm{~kb}\right)$

\section{Abbreviations}

CCK8: Cell counting kit-8; CSR: Class switch recombination;

FACS: Fluorescence-activated cell sorting; Ig: Immunoglobulin; LPS: Lipopolysaccharide; MM: Multiple myeloma; PBs: Plasmablasts; PCS: Plasma cells; SHM: Somatic hypermutation

\section{Acknowledgements \\ Not applicable.}

\section{Authors' contributions}

$Y F, R X, B Z$ and $C H$ performed the experiments. NM, LW, GH, ZJ and RW contributed essential reagents and materials for the experiments. RW conceived and designed the studies. YF and RW contributed to data analysis and manuscript preparation. All authors have read and approved the final manuscript.

\section{Funding}

This study was supported by grants from National Nature and Science Funds (31770956 and 81471529) and Beijing Natural Science Foundation (7182121). The funding bodies had no role in the design of the study and collection, analysis, and interpretation of data and in writing the manuscript. 


\section{Availability of data and materials}

The generated and/or analyzed datasets of the current study are available in the ArrayExpress repository, http://www.ebi.ac.uk/arrayexpress/experiments/EMTAB-7136.

\section{Ethics approval and consent to participate}

The study (No: 70956) was reviewed and approved by both the institutional committee and the animal ethics committee of Beijing Institute of Basic Medical Sciences.

\section{Consent for publication}

Not applicable.

\section{Competing interests}

The authors declare that they have no competing interests.

\section{Author details}

'Department of Rheumatology, First Hospital of Jilin University, Changchun 130021, China. ${ }^{2}$ Laboratory of Immunology, Institute of Basic Medical Sciences, P.O. Box 130 (3), Taiping Road \#27, Beijing 100850, China. ${ }^{3}$ College of Life Science and Technology, Xinjiang University, Urumqi 830046, Xinjiang, China. ${ }^{4}$ Department of Geriatric Hematology, Chinese PLA General Hospital, Beijing 100853, China.

Received: 19 September 2018 Accepted: 18 June 2019

Published online: 16 July 2019

\section{References}

1. Mahtouk K, Hose D, Reme T, De Vos J, Jourdan M, Moreaux J, et al. Expression of EGF-family receptors and amphiregulin in multiple myeloma Amphiregulin is a growth factor for myeloma cells. Oncogene. 2005;24: 3512-24.

2. Bataille R, Harousseau JL. Multiple myeloma. N Engl J Med. 1997;336:1657-64

3. Shapiro VS, Mollenauer MN, Weiss A. Endogenous CD28 expressed on myeloma cells up-regulates interleukin-8 production: implications for multiple myeloma progression. Blood. 2001;98:187-93.

4. Mahtouk K, Cremer FW, Reme T, Jourdan M, Baudard M, Moreaux J, et al Heparan sulphate proteoglycans are essential for the myeloma cell growth activity of EGF-family ligands in multiple myeloma. Oncogene. 2006;25: 7180-91.

5. Kumar A, Loughran T, Alsina M, Durie BG, Djulbegovic B. Management of multiple myeloma: a systematic review and critical appraisal of published studies. Lancet Oncol. 2003;4:293-304.

6. Tremblay-LeMay R, Rastgoo N, Chang H. Modulating PD-L1 expression in multiple myeloma: an alternative strategy to target the PD-1/PD-L1 pathway. J Hematol Oncol. 2018;11:46.

7. Kumar SK, Lee JH, Lahuerta JJ, Morgan G, Richardson PG, Crowley J, et al. Risk of progression and survival in multiple myeloma relapsing after therapy with IMiDs and bortezomib: a multicenter international myeloma working group study. Leukemia. 2012;26:149-57.

8. Epstein J, Hoover R, Kornbluth J, Barlogie B. Biological aspects of multiple myeloma. Baillierres Clin Haematol. 1995:8:721-34.

9. Nutt SL, Hodgkin PD, Tarlinton DM, Corcoran LM. The generation of antibodysecreting plasma cells. Nat Rev Immunol. 2015:15:160-71.

10. Shi W, Liao Y, Willis SN, Taubenheim N, Inouye M, Tarlinton DM, et al. Transcriptional profiling of mouse $B$ cell terminal differentiation defines a signature for antibody-secreting plasma cells. Nat Immunol. 2015;16:663-73.

11. Rossi M, Botta C, Arbitrio M, Grembiale RD, Tagliaferri P, Tassone P. Mouse models of multiple myeloma: technologic platforms and perspectives. Oncotarget. 2018:9:20119-33.

12. Spets H, Stromberg T, Georgii-Hemming P, Siljason J, Nilsson K, JernbergWiklund $\mathrm{H}$. Expression of the bcl-2 family of proand anti-apoptotic genes in multiple myeloma and normal plasma cells: regulation during interleukin6(IL-6)-induced growth and survival. Eur J Haematol. 2002;69:76-89.

13. Flores-Montero J, de Tute R, Paiva B, Perez JJ, Bottcher $\mathrm{S}$, Wind $\mathrm{H}$, et al. Immunophenotype of normal vs. myeloma plasma cells: toward antibody panel specifications for mrd detection in multiple myeloma. Cytometry B Clin Cytom. 2016;90:61-72.

14. Roy P, Sarkar UA, Basak S. The NF-kB activating pathways in multiple myeloma. Biomedicines. 2018;6:59.
15. Gonzalez D, van der Burg M, Garcia-Sanz R, Fenton JA, Langerak AW, Gonzalez $\mathrm{M}$, et al. Immunoglobulin gene rearrangements and the pathogenesis of multiple myeloma. Blood. 2007;110:3112-21.

16. Zhu G, Wang X, Xiao H, Liu X, Fang Y, Zhai B, et al. Both Notch1 and its ligands in B cells promote antibody production. Mol Immunol. 2017;91:17-23.

17. Wang X, Wei Y, Xiao H, Liu X, Zhang Y, Han G, et al. Pre-existing CD19independent GL7- Breg cells are expanded during inflammation and in mice with lupus-like disease. Mol Immunol. 2016;71:54-63.

18. Xu R, Fang Y, Hou C, Zhai B, Jiang Z, Ma N, et al. BC094916 suppressed SP 2/0 xenograft tumor by down?Regulating Creb1 and Bcl2 transcription. Cancer Cell Int. 2018;18:138.

19. Liu X, Zhang Y, Wang Z, Wang X, Zhu G, Han G, et al. Metabotropic glutamate receptor 3 is involved in B-cell-related tumor apoptosis. Int J Oncol. 2016;49:1469-78.

20. Zhu G, Liu X, Fang Y, Zhai B, Xu R, Han G, et al. Increased mTOR cancels out the effect of reduced Xbp-1 on antibody secretion in IL-1a-deficient B cells. Cell Immunol. 2018:328:9-17.

21. Ma N, Liu X, Xing C, Wang X, Wei Y, Han G, et al. Ligation of metabotropic glutamate receptor 3 (Grm3) ameliorates lupus-like disease by reducing B cells. Clin Immunol. 2015;160:142-54.

22. Ozcan E, Garibyan L, Lee JJ, Bram RJ, Lam KP, Geha RS. Transmembrane activator, calcium modulator, and cyclophilin ligand interactor drives plasma cell differentiation in LPS-activated B cells. J Allergy Clin Immunol. 2009;123:1277-86.

23. Calame KL, Lin Kl, Tunyaplin C. Regulatory mechanisms that determine the development and function of plasma cells. Annu Rev Immunol. 2003;21:205-30.

24. Shapiro-Shelef M, Lin Kl, McHeyzer-Williams L, Liao J, McHeyzer-Williams MG, Calame K. Blimp-1 is required for the formation of immunoglobulin secreting plasma cells and pre-plasma memory B cells. Immunity. 2003;19:607-20.

25. Clarke R, Cook KL, Hu R, Facey CO, Tavassoly I, Schwartz JL, et al. Endoplasmic reticulum stress, the unfolded protein response, autophagy, and the integrated regulation of breast cancer cell fate. Cancer Res. 2012;72 1321-31.

26. Clarke R, Shajahan AN, Wang Y, Tyson JJ, Riggins RB, Weiner LM, et al. Endoplasmic reticulum stress, the unfolded protein response, and gene network modeling in antiestrogen resistant breast cancer. Horm Mol Biol Clin Invest. 2011;5:35-44.

27. Palumbo A, Anderson K. Multiple myeloma. N Engl J Med. 2011;364:1046-60.

28. Ria R, Reale A, Vacca A. Novel agents and new therapeutic approaches for treatment of multiple myeloma. World J Methodol. 2014;4:73-90.

29. Borrello I. Can we change the disease biology of multiple myeloma? Leuk Res. 2012;36:S3-12.

30. Novak AJ, Darce JR, Arendt BK, Harder B, Henderson K, Kindsvogel W, et al. Expression of BCMA, TACl, and BAFF-R in multiple myeloma: a mechanism for growth and survival. Blood. 2004;103:689-94.

31. Lin FR, Huang SY, Hung KH, Su ST, Chung $\mathrm{CH}$, Matsuzawa A, et al. ASK1 promotes apoptosis of normal and malignant plasma cells. Blood. 2012;120:1039-47.

32. Shapiro-Shelef M, Calame K. Plasma cell differentiation and multiple myeloma. Curr Opin Immunol. 2004;16:226-34.

33. Letai A. Pharmacological manipulation of $\mathrm{BCl}-2$ family members to control cell death. J Clin Invest. 2005;115:2648-55.

34. Chauhan D, Catley L, Li G, Podar K, Hideshima T, Velankar M, et al. A novel orally active proteasome inhibitor induces apoptosis in multiple myeloma cells with mechanisms distinct from Bortezomib. Cancer Cell. 2005:8:407-19.

35. Catz SD, Johnson JL. Transcriptional regulation of bcl-2 by nuclear factor kB and its significance in prostate cancer. Oncogene. 2001;20:7342-51.

36. Cory S, Adams JM. The Bcl2 family: regulators of the cellular life-or-death switch. Nat Rev Cancer. 2002;2:647-56.

37. Pezzella F, Turley H, Kuzu I, Tungekar MF, Dunnill MS, Pierce CB, et al. BCl-2 protein in non-small-cell lung carcinoma. N Engl J Med. 1993;329:690-4.

38. Cleary M, Smith S, Sklar J. Cloning and structural analysis of cDNAs for bcl-2 and a hybrid bcl-2/immunoglobulin transcript resulting from the $t(14 ; 18)$ translocation. Cell. 1986:47:19-28.

39. Chand HS, Montano G, Huang X, Randell SH, Mebratu Y, Petersen H, et al. A genetic variant of $\mathrm{p} 53$ restricts the mucous secretory phenotype by regulating SPDEF and BCl-2 expression. Nat Commun. 2014;5:5567.

40. Gomez BP, Riggins RB, Shajahan AN, Klimach U, Wang A, Crawford AC, et al. Human X-box binding protein-1 confers both estrogen independence and antiestrogen resistance in breast cancer cell lines. FASEB J. 2007;21:4013-27

41. Martins G, Calame K. Regulation and functions of Blimp-1 in T and B lymphocytes. Annu Rev Immunol. 2018;26:133-69. 
42. Crotty S, Johnston RJ, Schoenberger SP. Effectors and memories: BCl-6 and Blimp-1 in T and B lymphocyte differentiation. Nat Immunol. 2010;11:114-20

43. Shapiro-Shelef M, Calame K. Regulation of plasma-cell development. Nat Rev Immunol. 2005:5:230-42.

44. Reimold AM, Iwakoshi NN, Manis J, Vallabhajosyula P, Szomolanyi-Tsuda E, Gravallese EM, et al. Plasma cell differentiation requires the transcription factor XBP-1. Nature. 2001;412:300-7.

45. Shaffer AL, Shapiro-Shelef M, Iwakoshi NN, Lee AH, Qian SB, Zhao H, et al. XBP1, downstream of Blimp-1, expands the secretory apparatus and other organelles, and increases protein synthesis in plasma cell differentiation. Immunity. 2004;21:81-93.

46. Lin FR, Kuo HK, Ying HY, Yang FH, Lin Kl. Induction of apoptosis in plasma cells by B lymphocyteinduced maturation protein-1 knockdown. Cancer Res. 2007;67:11914-23.

\section{Publisher's note}

Springer Nature remains neutral with regard to jurisdictional claims in published maps and institutional affiliations.

Ready to submit your research? Choose BMC and benefit from:

- fast, convenient online submission

- thorough peer review by experienced researchers in your field

- rapid publication on acceptance

- support for research data, including large and complex data types

- gold Open Access which fosters wider collaboration and increased citations

- maximum visibility for your research: over $100 \mathrm{M}$ website views per year

At BMC, research is always in progress.

Learn more biomedcentral.com/submissions 